\title{
RESEARCHPAPER
}

\section{Assessment of genetic purity in hybrid lines by field grow-out test and molecular markers}

\author{
VILAS JADHAV AND O.P. VERMA \\ Department of Molecular and Cellular Engineering, Jacob School of Biotechnology and Bio-Engineering, Sam \\ Higginbottom Institute of Agriculture, Technology and Sciences, ALLAHABAD (U.P.) INDIA \\ Email : om.verma@shiats.edu.in
}

Article Info :Received : 22.12.2015; Revised : 16.01.2016; Accepted : 10.02.2016

Tomato is a significant vegetable crop of special economic importance, ranking second to potato in the world. As genetic composition of a variety is its ultimate identity, achieving and maintaining genetic purity in crops is an important both from agronomic as well as breeding point of view. The present investigation was carried out to identify polymorphic primers where it can distinguish the hybrid parental line and utilization of this marker for further genotyping in the hybrid population. Towards this, 18 random decamer primers (RAPD), were screened between hybrid 2 parental lines, out of which only one primer (OPAO9) showed polymorphism and also study the tomato hybrid 2 parental lines consisted of 42 plants were analysed by using SSR Marker and only one primer (TGS0100) was found to be polymorphic which showed difference in banding pattern with hybrid 2 parental lines (male and female) and to check the genetic purity through protein analysis in the given tomato hybrid 2 parental lines (male and female). It was successfully reproduced in the hybrids. Thus, the present investigation shows the efficiency of RAPD and SSR marker as a molecular tool for genetic purity analysis. Hence, this marker was employed in the hybrid population. Furthermore DUS characters were also taken in the hybrids and parental lines. The results were correlating with the molecular data. Therefore, molecular GOT can be effectively employed in hybrid lots for purity checking. This could be better and fast technology than field GOT and it will be more useful for the quality assessment in the seed industries.

Key words : Parental line, Genetic purity, RAPD, SSR marker, Biochemical marker

How to cite this paper : Jadhav, Vilas and Verma, O.P. (2016). Assessment of genetic purity in hybrid lines by field grow-out test and molecular markers. Asian J. Bio. Sci., 11 (1) : 106-118 [Special Issue of AFBSAH-2016]. 